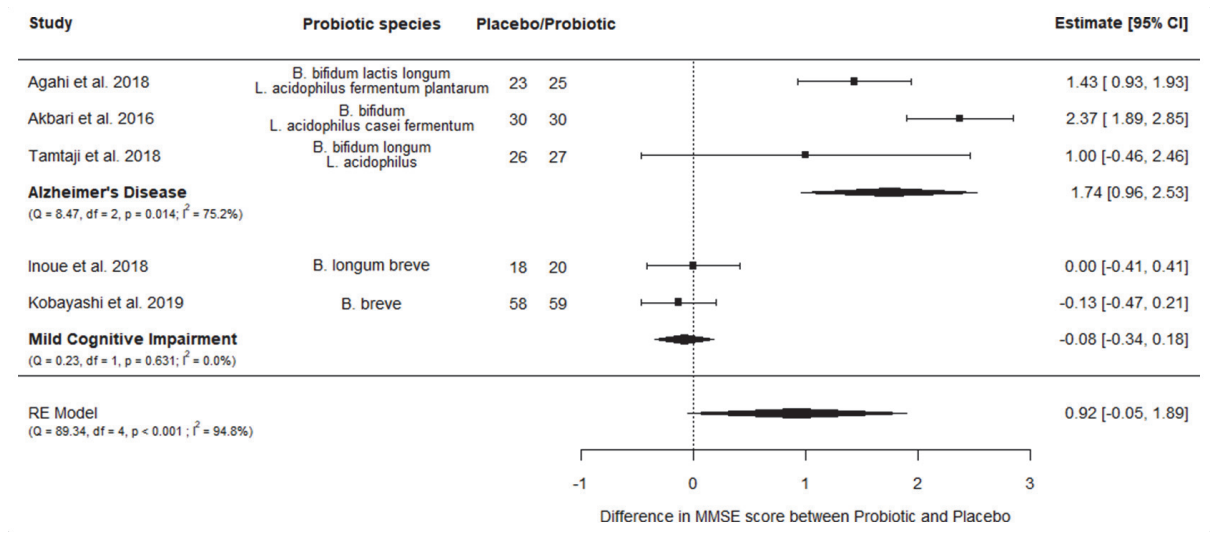

Abstract IDDF2020-ABS-0202 Figure 1 Forest Plot of MMSE scores

0.9 mark (0.1 to 1.9) improvement in MMSE scores in human RCTs, though the results are quite heterogeneous $\left(\mathrm{I}^{2}\right.$ $=94 \%$ ) (figure 1). Subgroup analysis of MCI and AD models were divergent with a difference of $-0.1(-0.3$ to 0.2$)$ versus a 1.7 (0.9 to 2.5) difference in MMSE score between the two groups. Studies also report improvement in other cognitive tests, such as CERAD and RBANS. Meta-regression revealed that the improvement in MMSE scores is age-dependent $(\mathrm{p}<$ 0.005 ) in humans. Biomarker analysis suggests that probiotic supplementation upregulates anti-oxidative ( $\downarrow$ MDA) and antiinflammatory ( $\downarrow$ hs-CRP) pathways. Studies also show an improvement in non-neurological symptoms such as in insulin sensitivity ( $\downarrow$ HOMA-IR, $\uparrow$ QUICKI), and lipid profiles $(\downarrow T G$, VLDL). However, an intervention study reported an increase in kynurenine:tryptophan ratio post probiotic supplementation, suggesting an activation of inflammatory pathways.

Conclusions Human study evidence generally shows an association between probiotic supplementation and improved neurocognitive function, although confounded by age and severity of neurodegeneration. Caution should be applied in the use of probiotics as an intervention for cognitive decline.

\section{IDDF2020-ABS-0203 INVESTIGATING THE EVIDENCE OF PREBIOTIC SUPPLEMENTATION IN THE ATTENUATION OF AGE-RELATED NEURODEGENERATION IN IN VIVO STUDIES: A SYSTEMATIC REVIEW AND META-ANALYSIS WITH BAYESIAN INFERENCE}

${ }^{1}$ Christopher Chi Hang Mak*, ${ }^{2}$ Henry Yue Hong Meng, ${ }^{3}$ Joyce Wing Yan Mak, ${ }^{4}$ Owen Ho Ko, ${ }^{5}$ Zuo Tao, ${ }^{3}$ Francis Ka Leung Chan. ${ }^{1}$ School of Clinical Medicine, University of Cambridge, UK; ${ }^{2}$ Faculty of Medicine, The Chinese University of Hong Kong, Hong Kong; ${ }^{3}$ Center for Gut Microbiota Research, Department of Medicine and Therapeutics, Institute of Digestive Disease, The Chinese University of Hong Kong, Hong Kong; ${ }^{4}$ Division of Neurology, Department of Medicine and Therapeutics, Faculty of Medicine, The Chinese University of Hong Kong, Hong Kong; ${ }^{5}$ Department of Medicine and Therapeutics, Institute of Digestive Disease, LKS Institute of Health Science, The Chinese University of Hong Kong, Hong Kong

\subsection{6/gutjnl-2020-IDDF.120}

Background Prebiotics, as non-digestible substances that stimulate the growth and activity of beneficial bacteria, is hypothesized to improve neurocognitive function through the Gut-

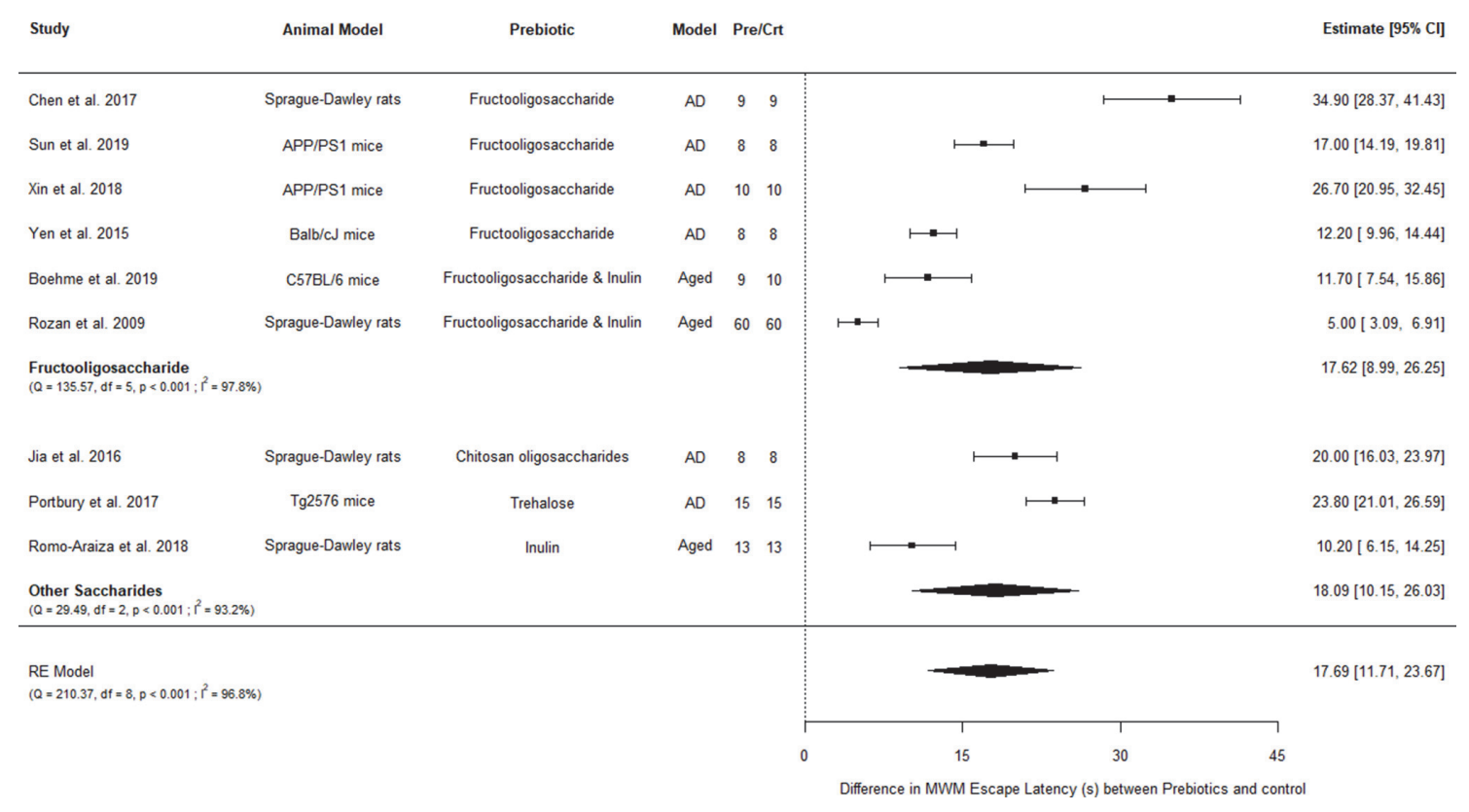

Abstract IDDF2020-ABS-0203 Figure 1 Forest plot of Morris Water Maze Escape Latency 
Brain axis. This review therefore, investigates whether the administration of prebiotics is efficacious in attenuating agerelated neurodegeneration.

Methods 2675 studies from MEDLINE, Embase, Scopus, Web of Science and Cochrane library were searched for in vivo studies using equivalent combinations of 'prebiotics' and 'agerelated neurodegeneration' in concordance with PRISMA guidelines. Quantitative outcomes such as Morris Water Maze (MWM), a cognitive-behavioural task, were examined and pooled with Forest Plots for overall effect (95\% CI) and heterogeneity $\left(\mathrm{I}^{2}\right)$. Weighted meta-regression of the prebiotic-neurodegeneration association with other continuous parameters such as treatment duration, study sample size and year of publication were assessed for potential confounding associations.

Risk of Bias (RoB) for animal studies was assessed by the SYRCLE tool. Publication bias was analysed by the BeggMazumdar funnel plot. The potential conflict of interest in the source of funding was examined by subgroup analysis.

Results 5 human studies on aged healthy volunteers were found. 23 animal studies were identified, with 9 animal studies having comparable quantitative results (149 subjects). Overall results in figure 1 demonstrate a $17.69 \mathrm{sec}$ (11.71-23.67; $I^{2}$ : 96.8) improvement in MWM Escape Latency, suggesting an improvement in neurocognitive function in animal models. Meta-regression revealed that prebiotic-neurodegeneration association is independent from duration of treatment $(p=0.202)$, year of publication $(p=0.184)$; and sample size $(p=0.0685)$.

RoB analysis of animal studies on prebiotics shows that there is a risk for bias in terms of study personnel blinding and random housing and outcome assessment of subjects. An asymmetric distribution that is largely beyond the 95\% CIs was observed in the Begg-Mazumdar funnel plot. 4 privatelyfunded studies had significantly weaker association of $12.67 \mathrm{~s}$ (3.18-22.17) than 5 government-funded studies $21.61 \mathrm{~s}$ (15.26-27.97).

Conclusions Weak evidence suggests that prebiotic supplementation is useful in attenuating age-related neurocognitive decline, thus requiring more clinical trials to evidence its true efficacy.

\section{IDDF2020-ABS-0207 EFFICACY AND SAFETY OF ENDOSCOPIC BALLOON DILATATION COMBINED WITH LOCAL INJECTION OF BETAMETHASONE IN THE TREATMENT OF INTESTINAL STENOSIS IN CROHN'S DISEASE}

${ }^{1}$ Junrong Chen*, ${ }^{2}$ Jiachen Sun, ${ }^{1}$ Xiang Peng, 'Tao Liu, ${ }^{1}$ Min Zhang, ${ }^{1}$ Min Zhi. 'Department of Gastroenterology, The Sixth Affiliated, Sun Yat-sen University, China; ${ }^{2}$ Department of Gastrointestinal Endoscopy, The Sixth Affiliated, Sun Yat-sen University, China

\subsection{6/gutjnl-2020-IDDF.121}

Background To evaluate the efficacy and safety of endoscopic balloon dilatation(EBD) combined with local injection of betamethasone in the treatment of intestinal stenosis in Crohn's disease(CD).

Methods A total of 51 patients with CD intestinal stenosis treated with EBD in the Sixth Affiliated Hospital of Sun Yatsen University from August 2013 to June 2020 were collected, including 38 patients treated with EBD alone and 13 patients treated with EBD combined with local injection of betamethasone. The efficacy and safety of the two treatment methods were compared.
Results Among the 51 patients, there were 36 males and 15 females, the median age was 32 years old, average body mass index (BMI) was 19.23, combined with the perianal disease was $54.9 \%$, follow-up time ranged from 0.4 to 78.6 months, and median follow-up time was 15.7 months. There was no significant difference in the disease course, activity, extraintestinal manifestations, perianal disease, endoscopic stenosis length, proximal CTE dilation diameter and other general information. In terms of treatment effect, there was no significant difference in treatment success rate $(100 \%$ vs. $94.7 \%, \mathrm{P}=1.000)$, symptom relief rate $(92.3 \%$ vs. $84.2 \%, \mathrm{P}=0.440)$ or complication rate $(0$ vs. $5.3 \%, \mathrm{P}=1.000)$ in the $\mathrm{EBD}$ combined with local injection of betamethasone. In the follow-up after treatment, it was found that the EBD combined with local injection of betamethasone group had fewer CD drug upgrades $(7.7 \%$ vs. $18.4 \%, \mathrm{P}=0.329)$, a lower rate of endoscopic expansion $(30.8 \%$ vs. $39.5 \%, \mathrm{P}=0.575)$, and a lower rate of surgery $(7.7 \%$ vs. $15.8 \%, \mathrm{P}=0.440)$.

Conclusions Ileocecal valve and colorectal anastomosis is the most common CD merger of the digestive tract stenosis position, EBD combined with local injections of betamethasone treatment of digestive tract stenosis type CD curative effect is reliable and safer. While this method on non-anastomotic stenosis, the history of intestinal fistula and the stenosis and dilation of nodular hyperplasia are not good, so it needs to be differentiated and selected in the treatment.

\section{IDDF2020-ABS-0208 FIRST-LINE UTILIZATION OF DIRECT PERORAL CHOLANGIOSCOPY FOR LARGE COMMON BILE DUCT STONES SAVES BOTH TIME AND COST}

Calvin Jianyi Koh*, Margaret Teng, Chieh Sian Koo, Bhavesh Doshi. National University Hospital, Singapore

\subsection{6/gutjnl-2020-IDDF.122}

Background Direct Peroral Cholangioscopy (POC) is a useful adjunct to facilitate lithotripsy in the management of large common bile duct stones during endoscopic retrograde cholangiopancreatograpy (ERCP) but has limitations due to its cost. In our centre, POC is limited to cases where anaesthesia care is available; this is not always the case due to resource constraints.

Methods We retrospectively evaluated our ERCP cases for large stone $(>10 \mathrm{~mm})$ and compared cases where POC was used as the first-line vs cases where 'standard of care' was performed. Besides demographics, indications and number of ERCPs cost and use of POC were considered.

Results In the 12-month period under consideration, a total of 46 cases met the inclusion criteria, with 6 cases of POC and 40 cases of standard ERCP. Cholangitis (47.8\%), and choledocholithiasis without cholangitis (45.7\%) accounted for most of the cases. Of the cases with first-line POC, 33.3\% required further ERCP, whereas $42.5 \%$ of the standard ERCP patients required another procedure for stone therapy. Due to the low numbers, these proportion differences did not reach statistical significance, although data collection is ongoing.

Of note, the cases with POC had a smaller average hospitalization bill size, $85.8 \%$ that of the standard ERCP. Although patients with POC had higher consumable fees, they had lower total hospitalization stay and hence had less total cost. (Figure 1). 